\title{
A Reliability Allocation Method of CNC Lathes Based on Copula Failure Correlation Model
}

Hao Wang ${ }^{1}$, Yi-Min Zhang ${ }^{2^{*}}$ and Zhou Yang ${ }^{1}$

\begin{abstract}
The current research of reliability allocation of CNC lathes always treat CNC lathes as independent series systems. However, CNC lathes are complex systems in the actual situation. Failure correlation is rarely considered when reliability allocation is conducted. In this paper, drawbacks of reliability model based on failure independence assumption are illustrated, after which, reliability model of CNC lathes considering failure correlation of subsystems is established based on Copula theory, which is an improvement of traditional reliability model of series systems. As the failure time of CNC lathes often obeys Weibull or exponential distribution, Gumbel Copula is selected to build correlation model. After that, a reliability allocation method considering failure correlation is analyzed based on the model established before. Reliability goal is set first and then failure rates are allocated to subsystems according to the allocation vector through solving the correlation model. Reliability allocation is conducted for $t=1$. A real case of a CNC lathe and a numerical case are presented together to illustrate the advantages of the reliability model established considering failure correlation and the corresponding allocation method. It shows that the model accords to facts and real working condition more, and failure rates allocated to all the subsystems are increased to some extent. This research proposes a reliability allocation method which takes failure correlation among subsystems of CNC lathes into consideration, and costs for design and manufacture could be decreased.
\end{abstract}

Keywords: CNC lathe, Copula, Failure correlation, Reliability model, Reliability allocation

\section{Introduction}

$\mathrm{CNC}$ lathes take an important role in mechanical equipment, the reliability technology of which is improved and developed gradually by scholars at home and abroad. Zhang et al. [1, 2] put forward the reliability syllabus of mechanical products including $\mathrm{CNC}$ lathes and researched the essence of reliability-based design. Wu et al. [3] presented a novel reliability assessment methodology with machining performance degradation data for estimating the reliability level of equipment. Li et al. [4] presented a reliability optimization design method considering the failure caused by overlarge static deformation of the $\mathrm{CNC}$ lathe spindle, so that reliability was

\footnotetext{
*Correspondence: ymzhang@mail.neu.edu.cn

${ }^{2}$ Equipment Reliability Institute, Shenyang University of Chemical

Technology, Shenyang 110142, China

Full list of author information is available at the end of the article
}

improved and reliability robustness increased at the same time. CNC lathes are traditionally regarded as series systems [5], and it's commonly seen in some researches that failures of different subsystems are independent, which means that failure of a certain subsystem cannot be influenced by any other subsystem. However, according to engineering experience and practical situation, failure independence hypothesis is not accurate enough [6], which may cause unnecessary costs. Problems such as changes in environment or uneven distribution of materials may lead to the same variation tendency of subsystems' reliability, resulting in fluctuation of the reliability of the lathe. Failure of a subsystem is not only related to fatigue caused by long time operation, but also related to failures of other systems, which is so-called failure correlation $[7,8]$. Therefore, failure correlation should be given full consideration when assessing reliability of CNC lathes. 
In recent years, many researches about failure correlation of different systems and structures have been conducted by scholars. Shen et al. [9] proposed an average maintenance time calculation method for $\mathrm{CNC}$ machine tools. Copula connection function was used to calculate failure rate function of components with failure correlation. Wang et al. [10] employed Gaussian Copula function to construct joint distribution of the random variables, and developed a novel model to describe the deterioration of aging structures in reliability analysis. Huang et al. [11] established avionics reliability assessment model based on Copula theory considering two kinds of failure modes. Mou et al. [12] researched reliability model of planetary steering gear using fault tree analysis and fuzzy theory. Zhang et al. [13] discussed failure correlation of $\mathrm{CNC}$ equipment taking $\mathrm{CNC}$ machine as an illustrative example, and compared the results with that of traditional series model. Chen et al. [14] proposed a reliability allocation method considering failure dependence, whose effect of cost reduction was demonstrated through a numerical case. Liu et al. [15] proposed a dimensionality reduction calculation method for multidimensional Copula, which enhanced computing efficiency to a large extent.

Based on the statement above, this paper established reliability model of $\mathrm{CNC}$ lathes considering failure correlation of subsystems using Copula theory, and then proposed a reliability allocation method based on the model. The rationality and effectiveness of the allocation method were explained and verified through two examples compared with traditional method based on failure independence assumption. Therefore, this paper provides reference and support for reliability design of CNC lathes.

\section{Copula Theory}

\subsection{Definition and Properties of Copula}

Copula function was first proposed by Sklar in 1959, which is a description for dependency relationship. It has been widely used in many fields, such as financial field, mechanical field and electronic field. Copula function can provide a link between joint distribution and marginal distribution of two or more random variables [16]. It can describe failure dependency or correlation roundly and reflect what's real of the relationships among different subsystems. Introducing Copula function into reliability analysis of $\mathrm{CNC}$ lathes can make up the shortage of traditional failure independence assumption.

Assuming that there are random variables, $x_{1}, x_{2}, \ldots, x_{n}$, the marginal distribution of which are respectively $F_{1}\left(x_{1}\right)$, $F_{2}\left(x_{2}\right), \ldots, F_{n}\left(x_{n}\right) . F_{i}\left(x_{i}\right)$ obeys uniform distribution between 0 and 1. Copula function that can describe these variables is written as $C\left(F_{1}\left(x_{1}\right), F_{2}\left(x_{2}\right), \ldots, F_{n}\left(x_{n}\right)\right)$. n-Dimensional Copula has the following characteristics [17].
(1) Domain of definition of $C\left(F_{1}\left(x_{1}\right), F_{2}\left(x_{2}\right), \ldots, F_{n}\left(x_{n}\right)\right)$ is $[0,1]^{n}=\boldsymbol{l}^{n}$.

(2) For any $F_{1}\left(x_{1}\right), \quad F_{2}\left(x_{2}\right), \ldots, \quad F_{n}\left(x_{n}\right) \in I^{n}, C\left(F_{1}\left(x_{1}\right)\right.$, $\left.F_{2}\left(x_{2}\right), \ldots, F_{n}\left(x_{n}\right)\right)$ is an increasing function.

(3) For any $F_{1}\left(x_{1}\right), F_{2}\left(x_{2}\right), \ldots, F_{n}\left(x_{n}\right) \in I^{n}$, if there exists $F_{i}\left(x_{i}\right)=0, C\left(F_{1}\left(x_{1}\right), F_{2}\left(x_{2}\right), \ldots, F_{n}\left(x_{n}\right)\right)=0$.

(4) For any $F_{1}\left(x_{1}\right), F_{2}\left(x_{2}\right), \ldots, F_{n}\left(x_{n}\right) \in I^{n}$, if all of them are equal to 1 except for $F_{i}\left(x_{i}\right), C\left(F_{1}\left(x_{1}\right), F_{2}\left(x_{2}\right), \ldots\right.$, $\left.F_{n}\left(x_{n}\right)\right)=F_{i}\left(x_{i}\right)$.

\subsection{Sklar Theorem}

It has been pointed out in Sklar theorem [18] that if joint distribution function of $n$-dimensional random variables is denoted as $F\left(x_{1}, x_{2}, \ldots, x_{n}\right)$, there exists a Copula function

$$
F\left(x_{1}, x_{2}, \ldots, x_{n}\right)=C\left(F_{1}\left(x_{1}\right), F_{2}\left(x_{2}\right), \ldots, F_{n}\left(x_{n}\right)\right),
$$

where if $F_{1}\left(x_{1}\right), F_{2}\left(x_{2}\right), \ldots, F_{n}\left(x_{n}\right)$ are continuous, $C\left(F_{1}\left(x_{1}\right)\right.$, $\left.F_{2}\left(x_{2}\right), \ldots, F_{n}\left(x_{n}\right)\right)$ is unique, otherwise, $C\left(F_{1}\left(x_{1}\right), F_{2}\left(x_{2}\right), \ldots\right.$, $\left.F_{n}\left(x_{n}\right)\right)$ is unique in $\operatorname{Ran} F_{1} \times \operatorname{Ran} F_{2} \times \cdots \times \operatorname{Ran} F_{n}$. According to the joint distribution function and marginal distribution function, and making $u_{i}=F_{i}\left(x_{i}\right)$, Eq. (1) can be rewritten as

$$
C\left(u_{1}, u_{2}, \ldots, u_{n}\right)=F\left(F_{1}^{-1}\left(x_{1}\right), F_{2}^{-1}\left(x_{2}\right), \ldots, F_{n}^{-1}\left(x_{n}\right)\right),
$$

where $F_{i}^{-1}\left(x_{i}\right)$ is the inverse function of $F_{i}\left(x_{i}\right)$.

According to Sklar theorem, $f\left(x_{1}, x_{2}, \ldots, x_{n}\right)$, which is the density function of $F\left(x_{1}, x_{2}, \ldots, x_{n}\right)$ can be written as

$$
\begin{aligned}
f\left(x_{1}, x_{2}, \ldots, x_{n}\right) \\
\quad=\frac{\partial F\left(x_{1}, x_{2}, \ldots, x_{n}\right)}{\partial x_{1} \partial x_{2} \ldots \partial x_{n}} \\
\quad=\frac{1}{2} \frac{\partial C\left(F_{1}\left(x_{1}\right), F_{2}\left(x_{2}\right), \ldots, F_{n}\left(x_{n}\right)\right)}{\partial x_{1} \partial x_{2} \cdots \partial x_{n}} \\
\quad=c\left(F_{1}\left(x_{1}\right), F_{2}\left(x_{2}\right), \ldots, F_{n}\left(x_{n}\right)\right) \times \prod_{i=1}^{n} f_{i}\left(x_{i}\right),
\end{aligned}
$$

where $c(\cdot)$ is the density function of Copula function, and $c\left(u_{1}, u_{2}, \ldots, u_{n}\right)=\frac{\partial C\left(u_{1}, u_{2}, \ldots, u_{n}\right)}{\partial u_{1} \partial u_{2} \cdots \partial u_{n}}, f_{i}\left(x_{i}\right)$ is the density function of marginal distribution function $F_{i}\left(x_{i}\right)$.

\subsection{Archimedean Copula Function}

There are many kinds of Copula functions, among which, Archimedean Copula function is widely used including Frank Copula, Clayton Copula and Gumbel Copula, and so on [19]. Expression of Archimedean Copula function is

$$
C_{A}\left(u_{1}, u_{2}, \ldots, u_{n}\right)=\varphi^{-1}\left(\varphi\left(u_{1}\right)+\varphi\left(u_{2}\right)+\cdots+\varphi\left(u_{n}\right)\right),
$$


where $0 \leq u_{i} \leq 1, \varphi$ is a non-increasing continuous function, called Copula generator. $\varphi:[0,+\infty) \rightarrow[0,1], \varphi$ $(0)=1, \lim _{x \rightarrow+\infty} \varphi(x)=0$, and $\varphi$ is strictly monotone decreasing in $\left[0, \varphi^{-1}(0)\right]$.

In mechanical systems, Gumbel Copula is mostly used, which is suitable for exponential distribution or Weibull distribution [11]. When the Copula generator $\phi\left(u_{i}\right)$ equals to $\left(-\ln u_{i}\right)^{1 / \theta}$, Gumbel Copula is formed, the expression of which is

$$
\begin{aligned}
C & \left(F_{1}\left(x_{1}\right), F_{2}\left(x_{2}\right), \ldots, F_{n}\left(x_{n}\right)\right) \\
= & \exp \left(-\left\{\left[-\ln \left(F_{1}\left(x_{1}\right)\right)\right]^{1 / \theta}+\left[-\ln \left(F_{2}\left(x_{2}\right)\right)\right]^{1 / \theta}\right.\right. \\
& \left.\left.+\cdots+\left[-\ln \left(F_{n}\left(x_{n}\right)\right)\right]^{1 / \theta}\right\}^{\theta}\right)
\end{aligned}
$$

where $\theta$ in $(0,1]$ is a parameter that describes correlation among variables. When $\theta \rightarrow 0$, it shows strong correlation, When $\theta=1$, there is no correlation among the variables. The estimated value $\hat{\theta}$ of $\theta$ can be obtained through maximum likelihood estimation method. Likelihood function $L(\theta)$ is just what in Eq. (3). $\hat{\theta}$ should make $L(\theta)$ reach its maximum, which can be expressed as $L\left(\hat{\theta} ; x_{1}, x_{2}, \ldots, x_{n}\right)=\max L\left(\theta ; x_{1}, x_{2}, \ldots, x_{n}\right) . \hat{\theta}$ can be obtained through solving $\frac{\partial C\left(u_{1}, u_{2}, \ldots, u_{n}\right)}{\partial \theta}=0$.

\section{Reliability Model of CNC Lathes Based on Copula Function}

$\mathrm{CNC}$ lathes are composed of several subsystems, denoted as $X_{1}, X_{2}, \ldots, X_{N}$. Assuming that lifetime of subsystems are $T_{1}, T_{2}, \ldots, T_{N}$, reliability of the $i$ th subsystem is
$R_{i}(t)=P\left(T_{i}>t\right)=1-F_{i}(t)$, where $F_{i}(t)$ is the unreliability of the $i$ th subsystem. According to independence assumption for series systems, reliability of the lathe is [20]

$$
R_{\mathrm{sys}}(t)=R_{1}(t) \cdot R_{2}(t) \cdots R_{N}(t) .
$$

To be more consistent with actual situation and give a more precise description of failure correlation among different subsystems, it's hypothesized that $n(n \leq N)$ subsystems are dependent in terms of failure, while the other $m(m=N-n)$ subsystems are independent. Reliability of failure dependent subsystems $\tilde{R}(t)$ can be calculated with Copula function. Assuming that the joint distribution function of lifetime of the $n$ subsystems with failure dependency is $H(t)=P\left(T_{1} \leq t, T_{2} \leq t, \ldots, T_{n} \leq t\right) . F_{i}(t)$ is the marginal distribution function of $T_{i}$, which is continuous. According to Sklar theorem, there exists the only $n$-dimensional Copula function and $H(t)=C\left(F_{1}(t), F_{2}(t)\right.$, ..., $\left.F_{n}(t)\right)$. Therefore, expression of $\tilde{R}(t)$ is

$$
\begin{aligned}
\tilde{R}(t)= & P\left(T_{1}>t, T_{2}>t, \ldots, T_{n}>t\right) \\
= & 1-\sum_{i=1}^{n} P\left(T_{i} \leq t\right)+\sum_{1 \leq j<k \leq n} P\left(T_{j} \leq t, T_{k} \leq t\right) \\
& +\cdots+(-1)^{m} \sum_{1 \leq i_{1}<i_{2}<\cdots<i_{m} \leq n} \\
& P\left(T_{i_{1}} \leq t, T_{i_{2}} \leq t, \ldots T_{i_{m}} \leq t\right) \\
& +\cdots+(-1)^{n} P\left(T_{1} \leq t, T_{2} \leq t, \ldots, T_{n} \leq t\right) .
\end{aligned}
$$

Equation (6) can be rewritten with Copula function as

$$
\begin{aligned}
\tilde{R}(t)= & C(1,1, \ldots, 1)-\sum_{i=1}^{n} C\left(1,1, \ldots, F_{i}(t), 1, \ldots, 1\right) \\
& +\sum_{1 \leq j<k \leq n} C\left(1,1, \ldots, F_{j}(t), 1, \ldots, F_{k}(t), 1, \ldots, 1\right)+\cdots+(-1)^{m} \\
& \times \sum_{1 \leq i_{i}<i_{2}<\cdots<i_{m} \leq n} C\left(1,1, \ldots, F_{i_{1}}(t), 1, \ldots, F_{i_{2}}(t), 1, \ldots, F_{i_{m}}(t), 1, \ldots, 1\right) \\
& +\cdots+(-1)^{n} C\left(F_{1}(t), F_{2}(t), \ldots, F_{n}(t)\right)=1-\sum_{i=1}^{n} F_{i}(t) \\
& +(-1)^{q} \sum_{1 \leq i_{1}<i_{2}<\cdots<i_{q} \leq n} C\left(1,1, \ldots F_{i_{1}}(t), 1, \ldots, F_{i_{2}}(t), \ldots, F_{i_{q}}(t), 1, \ldots, 1\right) \\
= & \Delta_{F_{1}(t)}^{1} \Delta_{F_{2}(t)}^{1} \ldots \Delta_{F_{n}(t)}^{1} C\left(F_{1}(t), F_{2}(t), \ldots, F_{n}(t)\right),
\end{aligned}
$$


where $\Delta$ is difference sign, and $\Delta_{a}^{b} \boldsymbol{\Theta}(x)=\boldsymbol{\Theta}(b)-\boldsymbol{\Theta}(a)$, in which $\Theta$ can be any function.

Finally, the failure dependent reliability model of the $\mathrm{CNC}$ lathe is obtained when considering failure correlation, which is

$$
\tilde{R}_{\text {sys }}(t)=\tilde{R}(t) \cdot \prod_{i=1}^{m} R_{i}(t) .
$$

It needs to explain that if there are $n_{1}$ subsystems with failure correlation, while other $n_{2}$ subsystems with failure correlation, and so on, then reliability model of the CNC lathe is that

$$
\begin{aligned}
\tilde{R}_{\text {sys }}(t) & =\tilde{R}_{n_{1}}(t) \cdot \tilde{R}_{n_{2}}(t) \cdots \tilde{R}_{n_{\sigma}}(t) \cdot \prod_{i=1}^{m} R_{i}(t) \\
& =\prod_{\gamma=1}^{\sigma} \tilde{R}_{n_{\gamma}}(t) \cdot \prod_{i=1}^{m} R_{i}(t),
\end{aligned}
$$

where $N_{1}, N_{2}, \ldots, N_{\sigma}$ are the numbers of subsystems with failure correlation respectively, the sum of which is $N-m$.

The upper bound of $\tilde{R}_{\text {sys }}$ is the result of the weakest link theory, denoted as $R_{\mathrm{w}}$, where $R_{w}=\min \left(R_{1}, R_{2}, \ldots, R_{N}\right)$. The lower bound of $\tilde{R}_{\text {sys }}$ is the result of independence hypothesis, denoted as $R_{\mathrm{p}}$, where $R_{\mathrm{p}}=\prod_{i=1}^{N} R_{i}$. So, there exists $R_{\mathrm{p}} \leq \tilde{R}_{\mathrm{sys}} \leq R_{\mathrm{w}}$. Assuming that there is a system including four failure dependent subsystems, reliability of which are respectively $R_{1}=0.9992, R_{2}=0.9796, R_{3}=0.9903$, $R_{4}=0.9888$. Then, $R_{\mathrm{p}}=R_{1} \times R_{2} \times R_{3} \times R_{4}=0.9585$, and $R_{\mathrm{w}}=\min \left(R_{1}, R_{2}, R_{3}, R_{4}\right)=0.9796$. Based on the statement above, reliability of the system using Copula function is

\section{A Reliability Allocation Method of CNC Lathes Considering Failure Correlation}

Reliability allocation is to allocate reliability index to products of each level from top to bottom according to certain principles and methods [21, 22]. Traditional ARINC allocation method [23] takes the failure rates of subsystems into consideration, which are used to calculate weight factors for allocation. The target failure rate is then allocated to each subsystem in proportion to the values of the weight factors. Wang et al. [24] considered seven factors, such as frequency and criticality of failure, and proposed a comprehensive allocation method for $\mathrm{CNC}$ lathes. Kim et al. [25] transformed the traditional severity level into exponential type to avoid the occurrence of unacceptable failure effects. Yadav et al. [26] proposed an allocation method through modifying traditional calculation method of criticality and introducing the potential for reliability improvement into allocation factor. In addition, Liu et al. [27] established a fuel cell vehicle model considering factors such as subsystem cost and limit reliability, and employed a genetic algorithm to reallocate the reliability of the overall vehicle system. Sriramdas et al. [28] evaluated allocation factors in linguistic terms and proposed an allocation approach.

All the methods mentioned above are based on independence hypothesis, however, failures of a subsystem are not only caused by fatigue and aging of the components, but also by failures of other subsystems. Many researchers have developed and improved the corresponding allocation methods. Zhuang et al. [29] proposed an allocation method using two-dimensional mapping of components and functions considering functional dependency and modified criticality factor. Yang et al. [30] gave an overall consideration of several allocation factors, established coefficient matrix of failure

$$
\begin{aligned}
\tilde{R}_{\text {sys }}(t)= & 1-\left(\sum_{i=1}^{4} F_{i}\right)+\sum_{1 \leq i<j \leq 4} \exp \left\{-\left[\left(-\ln F_{i}\right)^{1 / \theta}+\left(-\ln F_{j}\right)^{1 / \theta}\right]^{\theta}\right\} \\
& -\sum_{1 \leq i<j<k \leq 4} \exp \left\{-\left[\left(-\ln F_{i}\right)^{1 / \theta}+\left(-\ln F_{j}\right)^{1 / \theta}+\left(-\ln F_{k}\right)^{1 / \theta}\right]^{\theta}\right\} \\
& +\exp \left\{-\left[\sum_{i=1}^{4}\left(-\ln F_{i}\right)^{1 / \theta}\right]^{\theta}\right\}
\end{aligned}
$$

The change situation of $\tilde{R}_{\text {sys }}(t)$ along with parameter $\theta$ is shown in Figure 1. It can be seen that reliability considering failure correlation is between the result of the weakest link theory and that of independence hypothesis. With the increase of $\theta$, the result is close to that of independence hypothesis. correlation, calculated correlated failure severities of subsystems, and finally allocated the target reliability value to each subsystem.

Above all, taking failure correlation of subsystems into consideration, this section proposed a reliability allocation method applying Copula theory based on the 


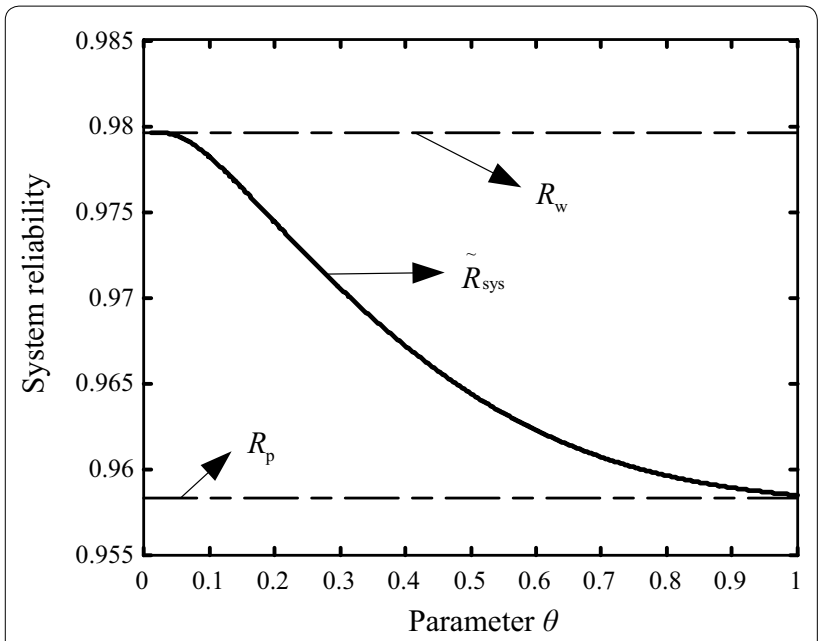

Figure 1 Changing curve of system reliability along with parameter $\theta$

reliability model established in Section 3. The final allocation failure rates of each subsystem are denoted as $\hat{\lambda}_{1}, \hat{\lambda}_{2}, \ldots, \hat{\lambda}_{N}$. It can be concluded from the allocation methods for series systems that there will be an allocation vector $\boldsymbol{A}=\left\{A_{1}, A_{2}, \ldots, A_{N}\right\}$. The target value of failure rate is allocated to each subsystem in accordance with $A$, so the failure rate allocated to the $i$ th subsystem is

$$
\hat{\lambda}_{i}=\frac{A_{i}}{\sum_{i=1}^{N} A_{i}} \lambda_{\mathrm{obj}},
$$

where $\lambda_{\mathrm{obj}}$ is the target value of failure rate that needed to be allocated to subsystems.

The following part focuses on allocation considering failure correlation. For all the $N$ subsystems, reliability allocation process is still carried out in accordance with the values in $\boldsymbol{A}$, which means that the ratio between failure rate of the $i$ th subsystem and that of the $j$ th subsystem is that $\lambda_{i}^{*}: \lambda_{j}^{*}=A_{i}: A_{j}$. Therefore,

$$
\lambda_{1}^{*}: \lambda_{2}^{*}: \cdots \lambda_{N}^{*}=A_{1}: A_{2}: \cdots: A_{N} .
$$

Failure time of $\mathrm{CNC}$ lathes usually obey exponential distribution or Weibull distribution. Here, reliability allocation is discussed taking exponential distribution as an example. Failure rate is constant for exponential distribution, and the relationship between reliability and failure rate is

$$
R_{i}^{*}(t)=e^{-\lambda_{i}^{*} t} .
$$

Reliability allocation is the primary stage of the life cycle of CNC lathes, which is conducted in this paper when $t=1$.

Setting the failure rate to be allocated to the $i$ th subsystem $\lambda_{i}^{*}$ as the standard, the failure rates to be allocated to other subsystems can be expressed with $\lambda_{i}^{*}$ according to Eq. (12). $\lambda_{i}^{*}$ can be solved through substituting Eq. (12) into the reliability model which is shown in Eq. (9), where, $F(t)=1-R(t)$, and letting Eq. (9) be equal to $e^{-\lambda_{\text {obj}}}$. Then, failure rates of the other $N-1$ subsystems can be obtained according to Eq. (12). Reliability results of each subsystem after allocation when $t=1$ are $R_{1}=e^{-\lambda_{1}^{*}}, R_{2}=e^{-\lambda_{2}^{*}}, \ldots, R_{N}=e^{-\lambda_{N}^{*}}$.

The following part illustrates the allocation method through an example, which is reliability allocation of a structure composed of four subsystems. Denoting observed failure rates as $\lambda_{1}, \lambda_{2}, \lambda_{3}$ and $\lambda_{4}$ respectively. For convenience, ARINC method is selected here, in which the allocation vector $\boldsymbol{A}=\left\{A_{1}: A_{2}: A_{3}: A_{4}=\lambda_{1}: \lambda_{2}: \lambda_{3}: \lambda_{4}\right\}$. Assuming that among all the four subsystems, $N_{1}, N_{2}$ and $N_{3}$ are failure dependent, and failure rate allocated to $N_{3}$ denoted as $\lambda_{3}^{*}$ is selected as the standard, then $\lambda_{1}^{*}=\frac{\lambda_{1}}{\lambda_{3}} \lambda_{3}^{*}$, $\lambda_{2}^{*}=\frac{\lambda_{2}}{\lambda_{3}} \lambda_{3}^{*}, \lambda_{4}^{*}=\frac{\lambda_{4}}{\lambda_{3}} \lambda_{3}^{*}$. Making $\theta=0.3$ for Gumbel Copula and Eq. (14) be equal to $e^{-\lambda_{\text {obj }}}, \lambda_{3}^{*}$ can be obtained by solving the equation.

$$
\begin{aligned}
\tilde{R}_{\text {sys }}= & \Delta_{F_{1}}^{1} \Delta_{F_{2}}^{1} \Delta_{F_{3}}^{1} C\left(F_{1}, F_{2}, F_{3}\right) \times R_{4} \\
= & \left\{1-\left(F_{1}+F_{2}+F_{3}\right)\right. \\
& +\exp \left(-\left[\left(-\ln \left(F_{1}\right)\right)^{(1 / 0.3)}+\left(-\ln \left(F_{2}\right)\right)^{(1 / 0.3)}\right]^{0.3}\right) \\
& +\exp \left(-\left[\left(-\ln \left(F_{1}\right)\right)^{(1 / 0.3)}+\left(-\ln \left(F_{3}\right)\right)^{(1 / 0.3)}\right]^{0.3}\right) \\
& +\exp \left(-\left[\left(-\ln \left(F_{2}\right)\right)^{(1 / 0.3)}+\left(-\ln \left(F_{3}\right)\right)^{(1 / 0.3)}\right]^{0.3}\right) \\
& -\exp \left(-\left[\left(-\ln \left(F_{1}\right)\right)^{(1 / 0.3)}+\left(-\ln \left(F_{2}\right)\right)^{(1 / 0.3)}\right.\right. \\
& \left.\left.\left.+\left(-\ln \left(F_{3}\right)\right)^{(1 / 0.3)}\right]^{0.3}\right)\right\} \times R_{4}=R_{\mathrm{obj}}=e^{-\lambda_{\mathrm{obj}}}, \\
& \left(F_{1}=1-R_{1}=1-e^{-\lambda_{1}^{*}}=1-e^{-\frac{\lambda_{1}}{\lambda_{3}} \lambda_{3}^{*}},\right. \\
& \left.F_{2}=1-e^{-\frac{\lambda_{2}}{\lambda_{3}} \lambda_{3}^{*}}, F_{3}=1-e^{-\lambda_{3}^{*}}, R_{4}=e^{-\frac{\lambda_{4}}{\lambda_{3}} \lambda_{3}^{*}}\right) .
\end{aligned}
$$

It can be concluded that failure rates allocated to subsystems are higher when considering failure correlation than that based on independence hypothesis, while the failure rate of the overall system remains the same, which still meets the design requirement. For a certain subsystem, if the acceptable failure rate is higher, then the cost of design and manufacture could be decreased, which makes it easier to make the subsystem up to standard. Therefore, the allocation method is more reasonable and consistent with engineering practice.

\section{Real Case and Discussions}

\subsection{Reliability Model of CNC Lathes Considering Failure Correlation}

This section establishes reliability calculation model of a $\mathrm{CNC}$ lathe using the theory in Section 3. The CNC lathe is divided into eight key subsystems such as main driving system (M), feeding system (F), cooling system (B), turret $(\mathrm{T})$, clamping system $(\mathrm{C})$, hydraulic system $(\mathrm{H})$, 
Table 1 Reliability functions and linear correlation coefficients of the subsystems

\begin{tabular}{lll}
\hline $\begin{array}{l}\text { Symbol } \\
\text { of subsystems }\end{array}$ & Reliability function & $\begin{array}{l}\text { Linear } \\
\text { correlation } \\
\text { coefficient } \boldsymbol{r}\end{array}$ \\
\hline $\mathrm{M}$ & $R_{\mathrm{M}}(t)=\exp \left[-(t / 2157.8)^{1.3739}\right]$ & 0.985 \\
$\mathrm{~F}$ & $R_{\mathrm{F}}(t)=\exp \left[-(t / 3184.8)^{1.7069}\right]$ & 0.956 \\
$\mathrm{~B}$ & $R_{\mathrm{B}}(t)=\exp \left[-(t / 2066.2)^{1.3727}\right]$ & 0.993 \\
$\mathrm{~T}$ & $R_{\mathrm{T}}(t)=\exp \left[-(t / 1971.0)^{1.2691}\right]$ & 0.982 \\
$\mathrm{C}$ & $R_{\mathrm{C}}(t)=\exp \left[-(t / 2415.7)^{1.5017}\right]$ & 0.949 \\
$\mathrm{H}$ & $R_{\mathrm{H}}(t)=\exp \left[-(t / 2279.9)^{1.2946}\right]$ & 0.987 \\
$\mathrm{E}$ & $R_{\mathrm{E}}(t)=\exp \left[-(t / 2410.2)^{2.2501}\right]$ & 0.984 \\
$\mathrm{P}$ & $R_{\mathrm{P}}(t)=\exp \left[-(t / 2435.2)^{2.1227}\right]$ & 0.985 \\
\hline
\end{tabular}

electric system (E) and protection system (P). Reliability functions (Weibull distribution as an example) and linear correlation coefficients are listed in Table 1. Reliability curves are shown in Figure 2.

When assuming that all the eight subsystems are failure independent, reliability model of the lathe is

$$
\begin{aligned}
& R_{\text {sys }}(t)=R_{\mathrm{M}}(t) R_{\mathrm{F}}(t) R_{\mathrm{B}}(t) R_{\mathrm{T}}(t) R_{\mathrm{C}}(t) R_{\mathrm{H}}(t) R_{\mathrm{E}}(t) R_{\mathrm{P}}(t) \\
&=\exp {\left[-\left(\frac{t}{2157.8}\right)^{1.3739}-\left(\frac{t}{3184.8}\right)^{1.7069}\right.} \\
&-\left(\frac{t}{2066.2}\right)^{1.3727}-\left(\frac{t}{1971.0}\right)^{1.2691} \\
&-\left(\frac{t}{2415.7}\right)^{1.5017}-\left(\frac{t}{2279.9}\right)^{1.2946} \\
&\left.-\left(\frac{t}{2410.2}\right)^{2.2501}-\left(\frac{t}{2435.2}\right)^{2.1227}\right]
\end{aligned}
$$

According to Ref. [13], subsystems with relatively low linear correlation coefficient are seen as failure dependent. From Table 1, correlation coefficients of feeding system $(\mathrm{F})$ and clamping system $(\mathrm{C})$ are 0.956 and 0.949 respectively, which are the lowest of all. Therefore, reliability model of the lathe is discussed in the following part considering failure correlation between feeding system (F) and clamping system (C).

According to Ref. [14], for systems like CNC lathes, Gumbel Copula is the most optimum choice to describe dependency among subsystems. Reliability model of the system including feeding system (F) and clamping system (C) is

$$
\begin{aligned}
\tilde{R}_{\mathrm{FC}}(t)= & \Delta_{F_{\mathrm{F}}(t)}^{1} \Delta_{F_{\mathrm{C}}(t)}^{1} C\left(F_{\mathrm{F}}(t), F_{\mathrm{C}}(t)\right) \\
= & 1-\left[1-R_{\mathrm{F}}(t)\right]-\left[1-R_{\mathrm{C}}(t)\right] \\
& +\exp \left\{-\left[\left(-\ln \left(1-R_{\mathrm{F}}(t)\right)\right)^{(1 / \theta)}\right.\right. \\
& \left.\left.+\left(-\ln \left(1-R_{\mathrm{C}}(t)\right)\right)^{(1 / \theta)}\right]^{\theta}\right\} .
\end{aligned}
$$

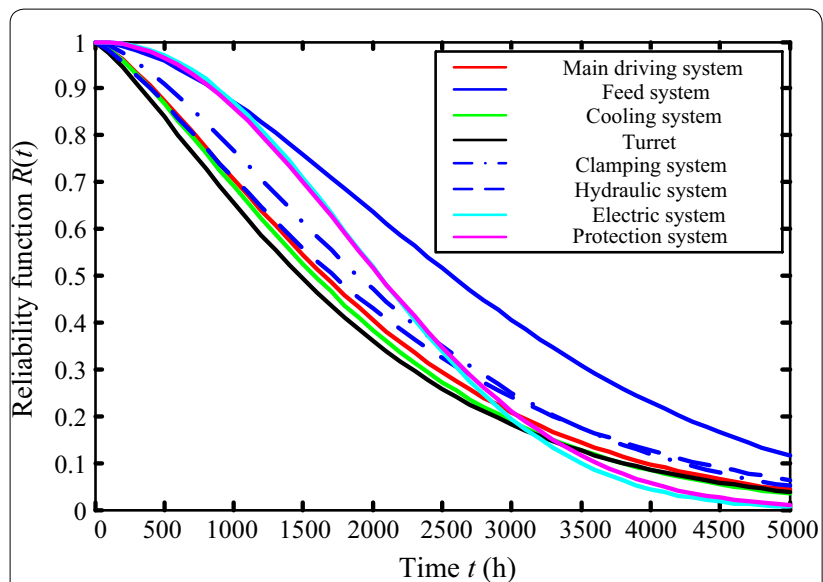

Figure 2 Changing curve of reliability of subsystems along with time

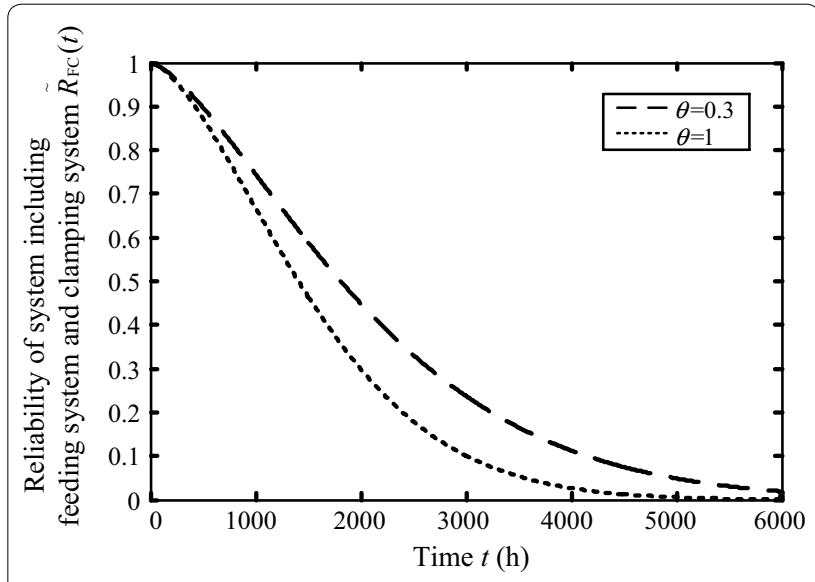

Figure 3 Changing situation of $\tilde{R}_{F C}(t)$ along with time

Changing situation of $\tilde{R}_{\mathrm{FC}}(t)$ along with time is shown in Figure 3 when parameter $\theta$ takes different values, where, feeding system (F) and clamping system (C) are independent when $\theta=1$. When $t$ remains unchanged, the smaller $\theta$ is, the stronger the correlation, and the higher the reliability will be. $\theta$ is set as 0.3 in this case.

To establish reliability model of the lathe, feeding system (F) and clamping system (C) are seen as an integrated system. Then, there are seven independent subsystems in series. Therefore, reliability model of the lathe when $\theta=0.3$ is

$$
\begin{aligned}
\tilde{R}_{\mathrm{Sys}}(t)= & \tilde{R}_{\mathrm{FC}}(t) \times R_{\mathrm{M}}(t) R_{\mathrm{B}}(t) R_{\mathrm{T}}(t) R_{\mathrm{H}}(t) R_{\mathrm{E}}(t) R_{\mathrm{P}}(t) \\
= & \Delta_{F_{\mathrm{F}}(t)}^{1} \Delta_{F_{\mathrm{C}}(t)}^{1} C_{0.3}\left(F_{\mathrm{F}}(t), F_{\mathrm{C}}(t)\right) \\
& \times R_{\mathrm{M}}(t) R_{\mathrm{B}}(t) R_{\mathrm{T}}(t) R_{\mathrm{H}}(t) R_{\mathrm{E}}(t) R_{\mathrm{P}}(t) \\
= & \left\{1-\left[1-R_{\mathrm{F}}(t)\right]-\left[1-R_{\mathrm{C}}(t)\right]\right. \\
& +\exp \left(-\left[\left(-\ln \left(1-R_{\mathrm{F}}(t)\right)\right)^{(1 / 0.3)}\right.\right. \\
& \left.\left.\left.+\left(-\ln \left(1-R_{\mathrm{C}}(t)\right)\right)^{(1 / 0.3)}\right]^{0.3}\right)\right\} \\
& \times R_{\mathrm{M}}(t) R_{\mathrm{B}}(t) R_{\mathrm{T}}(t) R_{\mathrm{H}}(t) R_{\mathrm{E}}(t) R_{\mathrm{P}}(t) .
\end{aligned}
$$




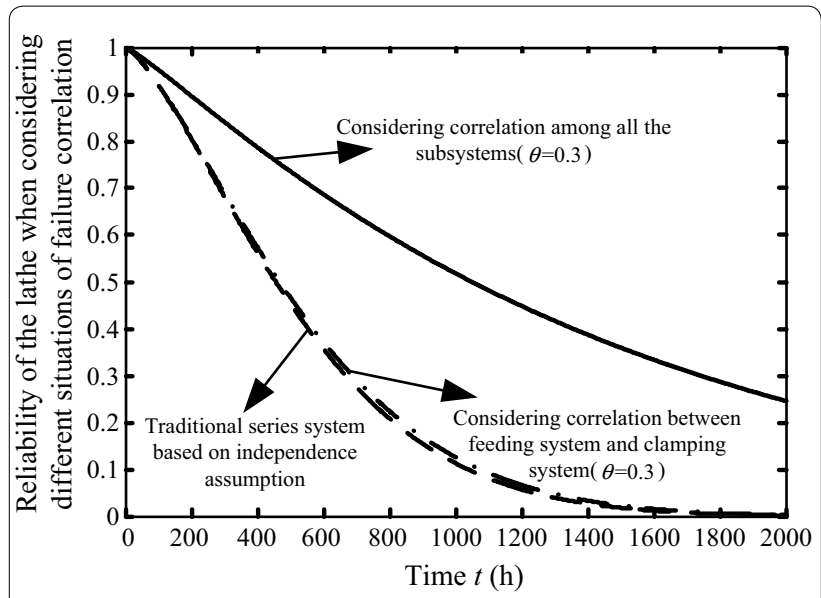

Figure 4 Reliability curves when considering different situations of failure correlation

Further, reliability model of the lathe considering failure correlation among all the subsystems is

$$
\begin{aligned}
\tilde{R}_{\text {sys }}(t)^{*}= & \Delta_{F_{\mathrm{M}(t)}}^{1} \Delta_{F_{\mathrm{F}(t)}}^{1} \Delta_{F_{\mathrm{B}(t)}}^{1} \Delta_{F_{\mathrm{T}(t)}}^{1} \Delta_{F_{\mathrm{C}(t)}}^{1} \Delta_{F_{\mathrm{H}(t)}}^{1} \Delta_{F_{\mathrm{E}(t)}}^{1} \Delta_{F_{\mathrm{P}(t)}}^{1} \\
& C_{0.3}\left(F_{\mathrm{M}}(t), F_{\mathrm{F}}(t), F_{\mathrm{B}}(t), F_{\mathrm{T}}(t),\right. \\
& \left.F_{\mathrm{C}}(t), F_{\mathrm{H}}(t), F_{\mathrm{E}}(t), F_{\mathrm{P}}(t)\right) .
\end{aligned}
$$

Figure 4 shows the reliability curves of the lathe when all the subsystems are independent, considering failure correlation between feeding system (F) and clamping system $(\mathrm{C})$ and considering failure correlation among all the subsystems. It can be seen from the figure that reliability of the lathe is the lowest when failure correlation is not considered, while reliability is becoming higher when considering failure correlation among more subsystems.

\subsection{Reliability Allocation of CNC Lathes}

Taking the data in Ref. [24] as a reference, an example of reliability allocation is presented by using the method in section 4, and comparison is made between the results of the method proposed in this paper and that of the method in Ref. [24]. Target value of failure rate $\lambda_{\text {obj }}=1 / M T B F_{\text {obj }}=0.002$, where, $M T B F_{\text {obj }}$ denotes the reliability goal of the overall CNC lathe in Ref. [24].

In Ref. [24], there were total 15 subsystems of CNC lathes and seven allocation factors are considered such as frequency of failure, criticality of failure, maintainability, complexity, manufacturing technology, working condition and cost. For each allocation factor, relative ratios for failure rate allocation between every two subsystems were calculated to get ratio matrix $\boldsymbol{B}^{(k)}$. Then, a matrix $\boldsymbol{\Gamma}$ can be obtained by calculating the average values of the entries in each row of the matrix $\boldsymbol{B}^{(k)}$ for the seven allocation factors. There is a vector $\boldsymbol{W}$ in Ref. [22] called weighting vector, representing the weight or importance
Table 2 Allocation results according to the method proposed in Ref. [24]

\begin{tabular}{llll}
\hline $\begin{array}{l}\text { Symbol } \\
\text { of subsystems }\end{array}$ & Subsystems & Failure rate $\hat{\boldsymbol{\lambda}}_{\boldsymbol{i}}$ & $\mathbf{M} \hat{\boldsymbol{T}} \boldsymbol{B} \boldsymbol{F}_{\boldsymbol{i}}(\mathbf{h})$ \\
\hline M & Turret & 0.000181 & 5523.56 \\
$J$ & Clamping accessory & 0.000163 & 6140.60 \\
V & Electric and electronic & 0.000141 & 7067.28 \\
& system & & \\
S1 & Main transmission & 0.000145 & 6890.52 \\
X & X feed system & 0.000158 & 6318.62 \\
Z & Z feed system & 0.000156 & 6398.46 \\
NC & CNC system & 0.000103 & 9679.27 \\
E & Power supply & 0.000123 & 8154.97 \\
D & Hydraulic system & 0.000138 & 7230.30 \\
F & Servo system & 0.000108 & 9222.45 \\
W & Cooling system & 0.000120 & 8305.45 \\
K & Swarf conveyors & 0.000135 & 7412.56 \\
L & Lubricant system & 0.000120 & 8346.89 \\
S2 & Spindle assembly & 0.000131 & 7645.18 \\
Q & Guard & 0.000076 & 13099.09 \\
\hline
\end{tabular}

of each allocation factor, which was obtained by expert rating method. The comprehensive failure rate allocation vector for all subsystems $\boldsymbol{A}$ was defined by $\boldsymbol{A}=\boldsymbol{W} \cdot \boldsymbol{\Gamma}$. Based on the data in Ref. [22], the allocation vector is finally obtained as follows:

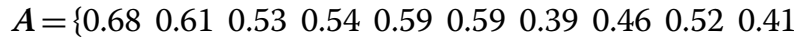
$0.450 .510 .450 .490 .29\}$.

Allocation results according to the method proposed in Ref. [24] are shown in Table 2, which are on the basis of independence hypothesis.

Considering failure correlation among all the 15 subsystems, target value of failure rate is still $\lambda_{\mathrm{obj}}=0.002$. Failure rate of Turret $(\mathrm{M}) \lambda_{1}^{*}$ is selected as the standard, and it can be obtained according to allocation vector $\boldsymbol{A}$ that

$$
\begin{aligned}
\lambda_{1}^{*}: \lambda_{2}^{*}: \cdots: \lambda_{15}^{*}= & 1: 0.8971: 0.7794: 0.7941: \\
& 0.8676: 0.8676: 0.5735: 0.6765: 0.7647: 0.6029: \\
& 0.6618: 0.7500: 0.6618: 0.7026: 0.4265
\end{aligned}
$$

where $\lambda_{i}^{*}$ denotes the failure rate to be allocated to the $i$ th subsystem by using the method proposed in this paper. Parameter $\theta$ of Gumbel Copula is taken as 0.3, and exponential distribution is considered for the failure time, reliability model of the lathe can be obtained

$$
\begin{aligned}
\tilde{R}_{\text {sys }}(t)^{*}= & \Delta_{F_{1(t)}}^{1} \Delta_{F_{2(t)}}^{1} \Delta_{F_{3(t)}}^{1} \cdots \Delta_{F_{15(t)}}^{1} \\
& \times C_{0.3}\left(F_{1}(t), F_{2}(t), F_{3}(t), \ldots, F_{15}(t)\right),
\end{aligned}
$$


Table 3 Allocation results considering failure correlation

\begin{tabular}{llll}
\hline $\begin{array}{l}\text { Symbol } \\
\text { of subsystems }\end{array}$ & Subsystems & Failure rate $\hat{\boldsymbol{\lambda}}_{\boldsymbol{i}}$ & $\mathbf{M T} \hat{\boldsymbol{B}}_{\boldsymbol{i}} \mathbf{h} \mathbf{h}$ \\
\hline M & Turret & 0.000263 & 3807.09 \\
J & Clamping accessory & 0.000236 & 4243.78 \\
V & Electric and electronic & 0.000205 & 4884.65 \\
& system & & \\
S1 & Main transmission & 0.000209 & 4794.22 \\
X & X feed system & 0.000228 & 4388.07 \\
Z & Z feed system & 0.000228 & 4388.07 \\
NC & CNC system & 0.000151 & 6638.35 \\
E & Power supply & 0.000178 & 5627.63 \\
D & Hydraulic system & 0.000201 & 4978.54 \\
F & Servo system & 0.000159 & 6314.63 \\
W & Cooling system & 0.000174 & 5752.63 \\
K & Swarf conveyors & 0.000197 & 5076.12 \\
L & Lubricant system & 0.000174 & 5752.63 \\
S2 & Spindle assembly & 0.000189 & 5283.23 \\
Q & Guard & 0.000112 & 8926.36 \\
\hline
\end{tabular}

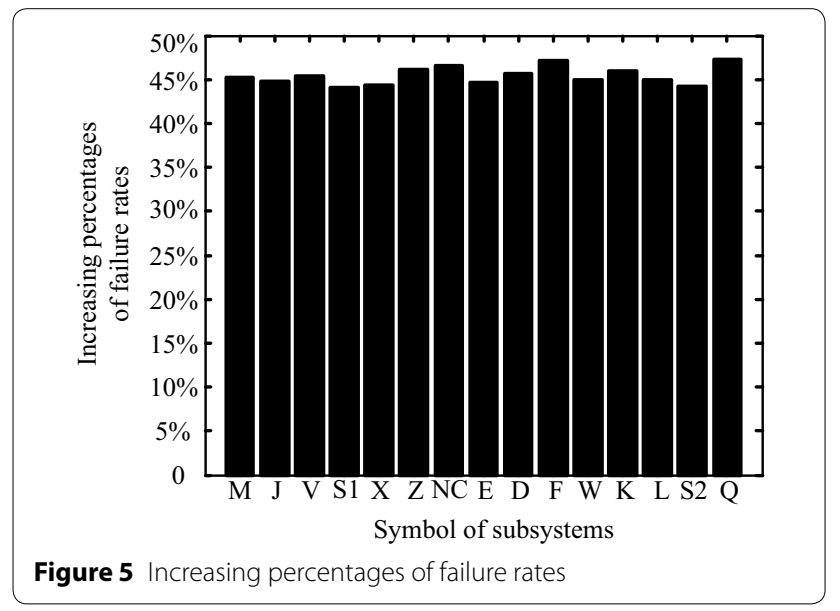

where $\quad F_{i}(t)=1-R_{i}(t)=1-e^{-\lambda_{i}(t)}$. Substituting Eq. (19) into Eq. (20) and letting Eq. (20) be equal to $R_{\text {obj }}=e^{-\lambda_{\text {obj }}}=e^{-0.002}(t=1)$, then $\lambda_{1}^{*}=0.000263$.

Allocation results are obtained according to Eq. (19) and $M T B F_{i}=1 / \lambda_{i}$, which are shown in Table 3 . It needs to state here that in this case, failure rate of Turret $(M)$ is selected as the calculation standard. In fact, failure rate of any other subsystem will be the choice for the standard, and it will not cause change to the allocation results.

The increasing percentages of failure rates when failure correlation is considered compared with that in Ref. [24] are shown in Figure 5. It can be concluded that failure rates of each subsystem have been increased by $40 \%$ to $50 \%$, while failure rate of the lathe is still 0.002 . Therefore, under the premise that MTBF of the lathe remains the same, reliability demands are lowered so that costs will be reduced to a large extent, which takes away unnecessary wastes and trouble for designers.

\section{Conclusions}

In this paper, a reliability model of $\mathrm{CNC}$ lathes considering failure correlation among subsystems is established by using Copula function, and a reliability allocation method is proposed based on the model. Reliability of failure dependent subsystems is first calculated, which is multiplied by reliability expressions of other systems that are failure independent so that reliability model of the lathe is obtained. A reliability allocation method considering failure correlation is proposed by using the allocation vector calculated in any method based on failure independence assumption and the model established before. From the theoretical analysis and the cases, the following conclusions can be drawn.

(1) CNC lathes are treated as failure dependent systems considering failure correlation among subsystems, which are described based on Copula theory. Gumbel Copula is applied to establish reliability model. By comparing the model established in this paper and that based on independence hypothesis, it can be concluded that failure correlation model can give a more precise description of the actual running state and increase estimated or predicted reliability of $\mathrm{CNC}$ lathes.

(2) Based on the established model, a reliability allocation method considering failure correlation is proposed. Ratios among failure rates to be allocated to each subsystem are obtained according to the allocation vector of any existing allocation method based on independence hypothesis, which are substituted into the model established before to get the final results. The allocated failure rates by the method in this paper are higher than that of the method based on independence hypothesis while the MTBF of the lathe remains the same, so that the advantage of the proposed method lies in that costs for design and manufacture can be reduced to a large extent.

(3) Through a numerical case applying the theory in this paper, practicability, rationality and accuracy of the method in this paper is verified. Therefore, the present study can be helpful to the reliability design and analysis of series systems like CNC lathes or other mechanical systems having similar structures. 


\section{Authors' Contributions}

Y-MZ was in charge of the whole trial; HW wrote the manuscript; ZY assisted with sampling and laboratory analyses. All authors read and approved the final manuscript.

\section{Author Details}

1 School of Mechanical Engineering and Automation, Northeastern University, Shenyang 110819, China. ${ }^{2}$ Equipment Reliability Institute, Shenyang University of Chemical Technology, Shenyang 110142, China.

\section{Authors' Information}

Hao Wang, born in 1991, is currently a PhD candidate at Mechanical Reliability and Dynamics Research Center, Northeastern University, China. His research interests include reliability design and reliability evaluation of mechanical systems.

Yi-Min Zhang, born in 1958, is currently a professor and PhD candidate supervisor at Equipment Reliability Institute, Shenyang University of Chemical Technology, China. He is also the Chang Jiang Scholar. His main research interests include mechanical reliability engineering and mechanical dynamic design.

Zhou Yang, born in 1979, is currently an associate professor at Mechanical Reliability and Dynamics Research Center, Northeastern University, China. Her research interests include mechanical reliability design and fluid dynamics.

\section{Competing Interests}

The authors declare that they have no competing interests.

\section{Funding}

Supported by National Natural Science Foundation of China (Grant Nos. 51135003, U1234208), National Basic Research Program of China (973 Program, Grant No. 2014CB046303), High-class CNC Machine Tools and Basic Manufacturing Equipment of Important National Science and Technology Specific Projects (Grant No. 2013ZX04011-011), National Key Laboratory of Mechanical System and Vibration Project (Grant No. MSV201402), Scientific Research Business Fund of Central Colleges and Universities (Grant No. N150304006), Excellent Talents Support Program for Colleges and Universities in Liaoning Province of China (Grant No. LJQ2014030).

\section{Publisher's Note}

Springer Nature remains neutral with regard to jurisdictional claims in published maps and institutional affiliations.

Received: 12 April 2017 Accepted: 26 November 2018

Published online: 27 December 2018

\section{References}

[1] YM Zhang. Connotation and development of mechanical reliabilitybased design. Journal of Mechanical Engineering, 2010, 46(14): 167-188. (in Chinese)

[2] Y M Zhang, Z L Sun. The reliability syllabus of mechanical products. Journal of Mechanical Engineering, 2014, 50(14): 14-20. (in Chinese)

[3] JWu, C Deng, XY Shao, et al. A reliability assessment method based on support vector machines for CNC equipment. Science in China Series E: Technological Sciences, 2009, 52(7): 1849-1857.

[4] CY Li, S Guo, Y M Zhang, et al. Reliability optimization design for stochastic static deformation of computer numerical control lathe spindle. Proceedings of the Institution of Mechanical Engineers, Part B: Journal of Engineering Manufacture, 2016, 230(1): 83-90.

[5] Z Yang, P X Liu, Y P Zhu, et al. A comprehensive reliability allocation method for series systems based on failure mode and effects analysis transformed functions. Proceedings of the Institution of mechanical Engineers, Part B: Journal of Engineering Manufacture, 2016, 230(12): 2239-2248

[6] Y Sun, L Ma, J Mathew, et al. An analytical model for interactive failures. Reliability Engineering \& System Safety, 2006, 91(5): 495-504.

[7] Y Cheng, X P Du. System reliability analysis with dependent component failures during early design stage - a feasibility study. Journal of Mechanical Design, 2016, 138(5): 051405
[8] Y Chen, L Yang, C Ye, et al. Failure mechanism dependence and reliability evaluation of non-repairable system. Reliability Engineering \& System Safety, 2015, 138: 273-283.

[9] GX Shen, W B Zeng, Y Z Zhang, et al. Determination of the average maintenance time of CNC machine tools based on type II failure correlation. Maintenance and Reliability, 2017, 19(4): 604-614.

[10] C Wang, H Zhang, Q W Li. Reliability assessment of aging structures subjected to gradual and shock deteriorations. Reliability Engineering \& System Safety, 2017, 161: 78-86.

[11] M S Huang, Q H Wang, Y H Li, et al. An approach for improvement of avionics reliability assessment based on copula theory. 9th International Conference on Reliability, Maintainability and Safety, Guiyang, China, June 12-15, 2011: 179-183.

[12] P B Mou, F H Tao, C Z Jia, et al. A copula-based function model in fuzzy reliability analysis on the planetary steering gear. International Conference on Quality, Reliability, Risk, Maintenance, and Safety Engineering, Chengdu, China, July 15-18, 2013: 375-378.

[13] Y Z Zhang, R Zheng, G X Shen, et al. Failure dependency of CNC equipment based on copula theory. Journal of Jilin University (Engineering and Technology Edition), 2011, 41(6): 1636-1640. (in Chinese)

[14] Z Z Chen, Y Liu, H Z Huang, et al. A reliability allocation method considering failure dependence. ASME 2013 International Design Engineering Technical Conferences and Computers and Information in Engineering Conference, Portland, USA, August 4-7, 2013: V008T12A006.

[15] Z Liu, F H Tao, C Z Jia. Reliability analysis of series system based on copula function. China Measurement \& Test, 2013, 39(1): 125-128. (in Chinese)

[16] R B Nelsen. An introduction to Copulas. 2nd ed. New York: Springer Press, 2006.

[17] X Li. Methods and applications of COPULA. Beijing: Economy \& Management Publishing House, 2014.

[18] A Sklar. Random variables, distribution functions, and copulas: a personal look backward and forward. Lecture Notes-Monograph Series, 1996, 28: $1-14$.

[19] Y P You, R Fang, X H Li. Allocating active redundancies to k-out-of-n reliability systems with permutation monotone component lifetimes. Applied Stochastic Models in Business and Industry, 2016, 32(5): 607-620.

[20] J Y Tang, Y X Zhao, P He, et al. Copula new theory for reliability calculation involving correlation in mechanical systems. Mechanical Science and Technology for Aerospace Engineering, 2009, 28(4): 532-535, 541. (in Chinese)

[21] T Chen, S L Zheng, HT Liao, et al. A multi-attribute reliability allocation method considering uncertain preferences. Quality and Reliability Engineering International, 2016, 32(7): 2233-2244.

[22] S Chatterjee, J B Singh, A Roy. A structure-based software reliability allocation using fuzzy analytic hierarchy process. International Journal of Systems Science, 2015, 46(3): 513-525.

[23] $\mathrm{K} \mathrm{H}$ Chang. A novel reliability allocation approach using the OWA tree and soft set. Annals of Operations Research, 2016, 244(1): 3-22.

[24] Y Q Wang, R Yam, M J Zuo, et al. A comprehensive reliability allocation method for design of CNC lathes. Reliability Engineering \& System Safety, 2001, 72(3): 247-252.

[25] K O Kim, Y Yang, M J Zuo. A new reliability allocation weight for reducing the occurrence of severe failure effects. Reliability Engineering \& System Safety, 2013, 117: 81-88.

[26] O P Yadav, X Zhuang. A practical reliability allocation method considering modified criticality factors. Reliability Engineering \& System Safety, 2014 , 129: 57-65.

[27] X T Liu, S L Zheng, J Z Feng, et al. Reliability reallocation for fuel cell vehicles based on genetic algorithm. Quality and Reliability Engineering International, 2015, 31(8): 1495-1502.

[28] V Sriramdas, S K Chaturvedi, H Gargama. Fuzzy arithmetic based reliability allocation approach during early design and development. Expert Systems with Applications, 2014, 41(7): 3444-3449.

[29] X Zhuang, S Limon, O P Yadav. Considering modified criticality factor and functional dependency for reliability allocation purposes. Proceedings of the 2014 Industrial and Systems Engineering Research Conference, Montreal, Canada, May 31-June 4, 2014: 2602-2611.

[30] Z Yang, Y P Zhu, Y M Zhang, et al. A comprehensive reliability allocation method for numerical-controlled lathes based on Copula function. ACTA Armamentarii, 2016, 37(1): 131-140. (in Chinese) 